\section{Platform for a Technological Leap in Antibiotics}

\section{Elsbeth Heinzelmann, science + technology journalist}

\section{Abstract: NTN Swiss Biotech ${ }^{\mathrm{TM}}$ brings together the Swiss} Biotech Association SBA, which is involved in regulatory, financial and legal issues, and biotechnet Switzerland, which is active in translational R\&D, to provide a technology base for joint projects. Biotechnet aims to push promising domains by creating topic-oriented platforms that enable academia and industry to work together to produce R\&D results of major importance to society and the economy. The first activity initiated by biotechnet is the Antibiotics Platform that has now been launched.

Keywords: Antimicrobial-resistant organisms · Gram-negative bacteria - Klebsiella pneumoniae - Multidrug-resistant strains · Pathogenesis of tuberculosis

Antimicrobial-resistant organisms are insidious: their rapid spread around the world in recent years represents a great risk for humanity. It seems that we can keep Gram-positive pathogens in check for the time being, but the increasing incidence of antibiotic-resistant Gram-negative infections is forcing us to accord high priority to combating this type of bacterial resistance.

\section{A Clear Objective in Mind}

"For multidrug-resistant (MDR) strains, in particular, there is often a lack of treatment options as they have become resistant to virtually all available antibiotics", explains Prof. Markus Seeger, research group leader in the Institute of Medical Microbiology at the University of Zurich. "Gram-negative bacteria are generally much less permeable to antibiotics and thus generally less susceptible to naturally occurring antibiotics. This renders the discovery of novel antibiotics very challenging but at the same time rewarding." He is the head of the Antibiotics Platform created by the NTN Swiss Biotech ${ }^{\mathrm{TM}}$ to combine the expertise of outstanding scientists from academia and industry in common projects supported by the Commission for Technology and Innovation (CTI). The reason for this step is that - following a period of stagnation - research and development in antibiotics are today globally in full swing. A key role in this domain is played by Switzerland with some highly innovative SMEs and start-ups that were unfortunately not well networked in the past. This has now changed with the Antibiotics Platform that involves all specialists within the sector to drive things forward both rapidly and efficiently.

In this close-knit network most industry partners have a clear emphasis on the discovery of antibiotics for the treatment of Klebsiella pneumoniae, Pseudomonas aeruginosa, Acinetobacter baumannii and Escherichia coli. These pathogens have become a major problem in the context of hospital-acquired (nosocomial) infections. In addition to these Gram-negative bacteria, multidrug-resistant Mycobacterium tuberculosis (MDRTB) - the causative agent of most cases of tuberculosis - has become a global threat. Members of the Antibiotics Platform from industry - among them BioVersys - and from academia - such as the microbiologist Stewart Thomas Cole, Head of the Cole Laboratory at EPFL - are working on the development of drugs to treat MDR-TB. In a joint start-up meeting in Bern in early autumn 2015, partners from academia and industry explained their views and objectives in forging plans for a common Antibiotics Platform.

\section{Everyone Contributes Individual Excellence}

The Center of Organic and Medicinal Chemistry (ZHW Waedenswil) places particular emphasis on the design and synthesis part of the drug discovery process. In order to improve the potency, selectivity and pharmacokinetic profiles of modulators of validated drug targets, the group rationally designs and synthesizes novel scaffolds and focused libraries based on X-ray data of co-crystal structures. "Our iterative process of computational design, organic synthesis and biological testing leads to detailed structure-activity relationships for therapeutically relevant drug targets", explains Prof. Rainer Riedl, head of the Center. "Our medical chemistry expertise allows for the development of clinical drug candidates for the treatment of bacterial infections."

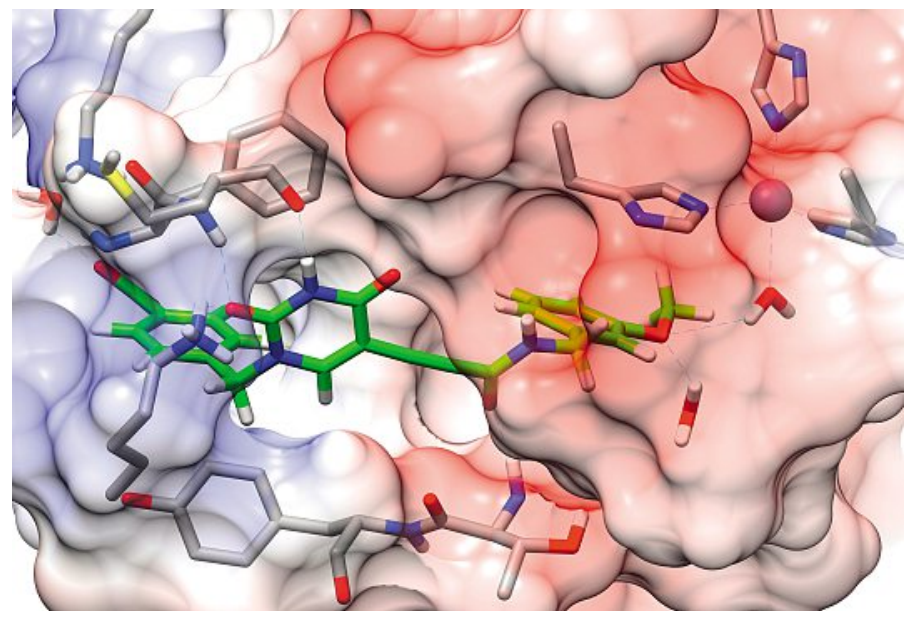

Structural understanding of the therapeutic target allows the rational design of optimized drug molecules. J. Lanz, R. Riedl, ChemMedChem 2015, 10, 451-454.

Prof. Stewart T. Cole is Director of the Global Health Institute at EPFL. As the Scientific Coordinator of three successful largescale EC Framework projects he has a track record of bilateral cooperation with academic institutions, SMEs and pharmaceutical companies. His world-class research unit is dedicated to TB drug discovery, unravelling the pathogenesis of tuberculosis and studying the phylogeography of leprosy. "I see a decisive trend towards more microbiome analyses and bioinformatics as well as sequence-driven research", says the microbiologist. "There is a major need in the context of rapid diagnostics. We could contribute to industrial partners of the platform the use of biosafety level III facilities that already exist at EPFL, in Bern and at UZH."

At the Laboratory of Molecular Evolution, University of Basel, the group working with Dr. Marc Creus is analyzing the 
evolution of resistance to antibiotics in Gram-negative bacteria by means of next-generation sequencing. As an example he cites a detailed clinical case report of a patient infected with a strain of Klebsiella pneumoniae, which developed multidrug antibiotic resistance during hospital treatment in Switzerland. In collaboration with an industrial partner, the Creus group has also applied similar sequencing technology to explore the potential adaptation of Gram-negative pathogens to a novel siderophore antibiotic that is currently undergoing clinical trials. "Exploring the evolution of resistance in the laboratory even before an antibiotic is marketed may be extremely informative in understanding the mechanism of action of the drug and may also help to minimize the evolution of resistance later on in the clinical setting."

The Institute for Chemistry \& Bioanalytics at the FHNW Muttenz is particularly active in the research fields biochemistry, bioanalytics \& diagnostics, bio-nanotechnology and organic synthesis \& chemical engineering.

The bioanalytics group contributed to BioVersys's CTI projects by providing kinetic binding parameters as indicators for lead identification and optimization. The crystallization of drugtarget complexes increases understanding of the binding pocket and allows the development of strategies towards the synthesis of better binders. Together with the organic synthesis group at the Institute, this comprehensive expertise in characterizing highly specific drug-target interactions using both kinetic and thermodynamic binding parameters with cutting-edge methodology forms a good basis for running integrated drug discovery projects. All the members of the organic synthesis group worked for many years in the pharmaceutical industry and thus provide very sound experience in medicinal chemistry, which has enabled the Institute to successfully run various projects in lead discovery and lead optimization both with small biotech and big pharmaceutical companies. In addition to this line of investigation, Prof. Eric Kuebler, whose focus is DNA analysis and mutagenesis, recently started a project with BioVersys to investigate potential resistance mechanisms related to BioVersys's anti-mycobacterial compounds.

The biotechnet has for several years had a close relationship with the Laboratory of Molecular Microbiology and Biotechnology run by Prof. Anna Maria Puglia at the University of Palermo.

\section{Rapid Exchange of Know-how}

SNSF Professor Markus Seeger is interested in the structure and function of antibiotic efflux. In particular, tripartite efflux such as the well-studied Acr/AcrB/TolC protein complex from Escherichia coli are major contributors to the drug-resistance in Gram-negative ESKAPE pathogens. But ABC transporters, which pump antibiotics out of the cell at the expense of ATP hydrolysis, are also mediators of intrinsic antibiotic resistance, mainly in Gram-positive cells. "We want to understand the molecular details of the pumping mechanisms that lead to antibiotic efflux", explains Markus Seeger. "For this purpose, we use Xray crystallography, antibiotic binding and transport studies and mutational analysis of residues lining the antibiotic efflux tunnel." Crystal structures determined by him and his group feature tunnels inside the protein and imply a 'peristaltic mode' of drug transport which could account for the wide substrate specificity observed.

Prof. Vincent Perreten at the Institute of Veterinary Bacteriology, University of Bern, performs epidemiological studies of antibiotic resistance using microarray technology and nextgeneration sequencing. This involves clinical samples from companion and food-producing animals, with monitoring of samples from healthy animals at the slaughterhouse and food samples of animal origin, i.e. meat.

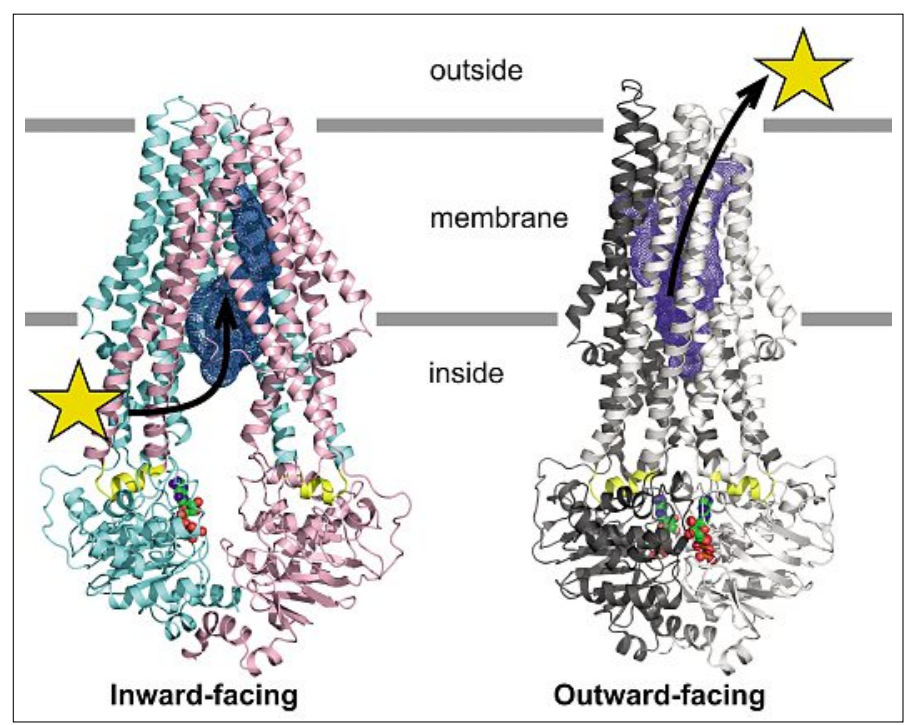

ABC transporters pump antibiotics out of the cell and thereby confer multidrug resistance. R. J. Dawson, K. P. Locher, Nature 2006, 443, 180-185. M. Hohl, C. Briand, M. G. Grütter, M. A. Seeger, Nat. Struct. Mol. Biol. 2012, 19, 395-402.

Dr. Daniel Obrecht, co-founder and CSO of Polyphor Ltd. in Allschwil, is focusing on the discovery and development of macrocycle drugs to address areas with a high level of unmet medical need. Currently, the company is seeking to develop novel antibiotics against MDR ESKAPE pathogens such as Klebsiella spp and Acinetobacter spp where multi-drug resistance is becoming a real threat. Further areas of interest are proteomic analyses of outer-membrane proteins in Gram-negative bacteria and photoaffinity labelling of antibiotics for target identification. "I see a major need in rapid diagnostics of Gram-negative bacteria in hospitals, including Pseudomonas infections", comments Daniel Obrecht. "A domain of more general interest is the effect of drugs on the host defence, in particular in the context of sepsis."

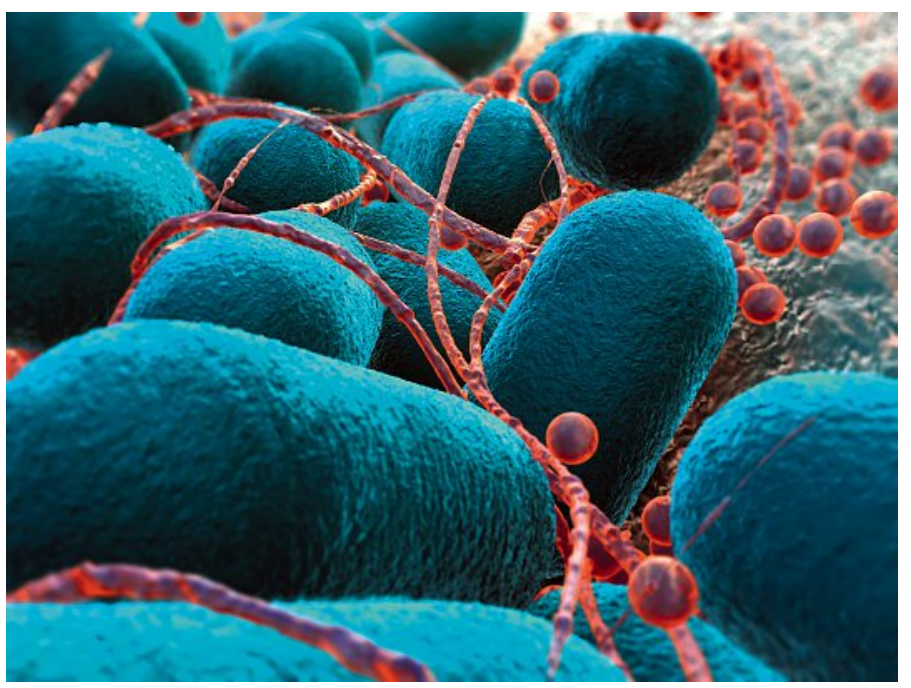

Electron micrograph of the Gram-negative ESKAPE pathogen Escherichia coli.

\section{A Platform of 'Give and Take'}

For years, Laves Arzneimittel GmbH in Schötz has concentrated on the exploration and development of preparations based on the Escherichia coli strain discovered in 1931 by Dr. Wolfgang Laves. Hans-Dieter Grimmecke, Head of R\&D, wants to under- 
stand the biofilm of the gut: "Probiotic bacteria of the future will probably originate from the gut microflora", he says. "A promising field of application for probiotics is the animal industry, in particular in the context of reducing or even banning antibiotics in livestock production."

As long ago as 2004, Actelion Pharmaceuticals Ltd. initiated a research programme in antibiotics, focusing on novel chemical scaffolds with new mechanisms of action against established targets. Goals are the intravenous treatment of severe hospital infections and oral antibiotics for community-acquired infections. "Drug transport - influx and efflux - is particularly interesting in the context of Gram-negative bacteria", explains Daniel Ritz, head of Anti-Infectives Biology. "They are much more difficult to treat than Gram-positive bacteria, mainly because of the presence of the outer membrane and the action of tripartite efflux pumps."

"Networking with experts in the field of bacterial infections is crucial for the advancement of antibacterial research and the development of novel antibiotics", says Dr. Juerg Dreier. He is group leader Biochemistry \& Screening and project leader of preclinical antibacterial projects at Basilea Pharmaceutica International Ltd. Basilea is focusing on the development of antibiotics, antifungals and oncology drugs. The company has a portfolio of commercial-stage drugs as well as a pipeline of innovative earlystage anti-infective and oncology product candidates. Through the integrated research, development and commercial operations of its Swiss subsidiary Basilea Pharmaceutica International Ltd., the company focuses on providing innovative pharmaceutical products in the therapeutic areas of bacterial infections, fungal infections and oncology, targeting the medical challenge of rising resistance and non-response to current treatment options.

BioVersys $A G$ in Basel develops small chemical molecules that switch off drug resistance on a gene-regulatory level within bacteria, known as TRICs (Transcriptional Regulator Inhibitory
Compounds). "We are working on an anti-tuberculosis project and several approaches to treating Gram-negative infections. We want to collaborate in the areas of X-ray crystallography and in silico modelling and obtain access to strain collections", says Dr. Michel Pieren, Group Leader Drug Discovery. "We have recently developed a novel technique to create targeted gene deletions in clinically relevant Gram-negative pathogens without the use of antibiotic markers. This is very useful when generating knockouts in multidrug-resistant Gram-negative bacteria." This method is of potential interest for other platform members.

\section{Remain Modest, Be Powerful!}

The platform members will now work out common areas of interest that are not affected by intellectual property issues. A priority seems to be the establishment of general methods to study plasma protein binding. "There is a strong commitment from academia and industry", Markus Seeger concludes. "We are not seeking to grow in terms of members, but want to realize a lean, effective organization with clear targets to produce visible results that receive international attention." For Prof. Daniel Gygax, President of biotechnet Switzerland, the clear aim is to increase competitiveness by creating interactive value: "Our focus is on linking the core competencies of companies with the scientific knowledge and experience of academia. We have to 'plug the holes' in know-how and technology along the entire value chain!"

Homepage: www.biotechnet.ch

Received: November 2, 2015 